\title{
Comorbidity amplifies the effects of post-9/11 posttraumatic stress disorder trajectories on health-related quality of life
}

\author{
Jiehui Li ${ }^{1} \cdot$ Kimberly Caramanica Zweig $^{1} \cdot$ Robert M. Brackbill $^{1} \cdot$ Mark R. Farfel $^{1} \cdot$ James E. Cone $^{1}$
}

Accepted: 12 December 2017 / Published online: 20 December 2017

(C) The Author(s) 2017. This article is an open access publication

\begin{abstract}
Purpose The present study aims to examine the impact of physical and mental health comorbidities on the association between post-9/11 posttraumatic stress disorder (PTSD) trajectories over 10 years and health-related quality of life (HRQOL) among 9/11-exposed persons.

Methods 30,002 responding adult World Trade Center Health Registry enrollees reporting no pre-9/11 PTSD were studied. PTSD trajectories (chronic, delayed, remitted, no PTSD) were defined based on a 17-item PTSD Checklist-Specific to 9/11 across three waves of survey data. Three indicators of poor HRQOL were defined based on CDC HRQOL-4 measures. We computed age-adjusted prevalence of physical and mental health comorbidity (depression/anxiety) by PTSD trajectory and used modified Poisson regression to assess the effect of PTSD trajectory on poor HRQOL prevalence, accounting for comorbidity.

Results Age-adjusted prevalence of overall comorbid conditions was 95.8 and $61.4 \%$ among the chronic and no-PTSD groups, respectively. Associations between 9/11-related PTSD trajectories and poor HRQOL were significant and became greater when comorbidity was included. Adjusted prevalence ratios were elevated for fair/poor health status (APR 7.3, 95\% CI $6.5,8.2), \geq 14$ unhealthy days $(4.7 ; 95 \%$ CI $4.4,5.1)$, and $\geq 14$ activity limitation days during the last 30 days $(9.6 ; 95 \%$ CI $8.1,11.4$ ) in the chronic PTSD group with physical and mental health comorbidity compared to those without PTSD and comorbidity; similar associations were observed for delayed PTSD.

Conclusions Ten years post-9/11 physical and mental health comorbidities have a substantial impact on the PTSD trajectories and HRQOL association. The need for early identification and treatment of PTSD and comorbidity should be emphasized to potentially improve HRQOL.
\end{abstract}

Keywords PTSD $\cdot$ Health-related quality of life $\cdot$ Comorbidity $\cdot$ World Trade Center

\section{Introduction}

The September 11, 2001 (9/11) terrorist attacks on the World Trade Center (WTC) in New York City (NYC) exposed hundreds of thousands of individuals to an immense cloud of dust and debris, numerous hazardous substances, and emotional trauma. Those with these experiences have an

Electronic supplementary material The online version of this article (https://doi.org/10.1007/s11136-017-1764-5) contains supplementary material, which is available to authorized users.

Jiehui Li

jli3@health.nyc.gov

1 New York City Department of Health and Mental Hygiene, World Trade Center Health Registry, 125 Worth Street, CN-6W, New York, NY 10013, USA increased risk of mental disorders and comorbid mental and physical health conditions. To prevent disability and optimize functioning and quality of life among those impacted by $9 / 11$ exposure, it is important to understand the burden of mental and physical conditions among the exposed population and to address the well-being of individuals with mental and/or physical health conditions long after the exposure.

Posttraumatic stress disorder (PTSD) is one of the most commonly reported mental health conditions following exposure to the $9 / 11$ disaster [1-6]. PTSD symptoms can last for many years [7]. Chronic PTSD symptoms among 9/11-exposed responders and civilians over 8-9 years of follow-up have been reported [8-10]. Pietrzak et al. reported that $5.3 \%$ of police and $9.5 \%$ of non-traditional responders experienced chronic PTSD, while $8.5 \%$ of police and $6.7 \%$ of non-traditional responders reported delayed-onset 
PTSD [8]. The WTC Health Registry reported similar findings: $4.0 \%$ chronic PTSD and $6.4 \%$ delayed-onset PTSD among rescue/recovery workers [9] and $8.0 \%$ chronic and $8.2 \%$ delayed-onset PTSD among civilians not involved in rescue/recovery work [10]. The effects of chronic post-9/11 PTSD on quality of life can be substantial due to long-lasting or re-occurring symptoms and increased risk of comorbid health conditions.

PTSD is often comorbid with other psychological disorders [11-13], as well as physical health conditions. Mental disorders of all kinds are associated with increasing risk of subsequent onset or diagnosis of a number of physical conditions, such as heart and lung diseases, diabetes, and arthritis [14]. The rates and burden of comorbidity are high in those with 9/11-related PTSD [5, 15-18]. The prevalence of depression among 9/11 responders with PTSD ranged from $66.5 \%$ [5] to $73.0 \%[15,16]$. The prevalence of anxiety among police 9/11 responders with PTSD was 53.5\% [13]. Among responders with PTSD, 70.0\% reported at least one of three aerodigestive disorders (asthma, sinusitis, and gastroesophageal reflux disease) [16]. Among civilian survivors, $40 \%$ of those with PTSD reported lower respiratory symptoms [17]. Nevertheless, the burden of comorbid physical health conditions in persons with 9/11-related PTSD may be greater if accounting for a wider range of physical health conditions beyond aerodigestive disorders [16-18].

When evaluating the burden of diseases, there has been increased focus on measuring the patient's perspective. Health-related quality of life (HRQOL) is a measure of an individual's perceived physical and mental health over time, and an indicator of disease burden. The adverse effects of PTSD [19], depression [20], anxiety [21], and physical health conditions [22] on HRQOL have been well documented in various populations. PTSD has been associated with reduced HRQOL [19], and may result in worse HRQOL in the presence of comorbid depression, anxiety, or physical health conditions [23, 24]. Individuals with PTSD and depression have worse QOL than those with PTSD alone [23]. There is evidence suggesting PTSD and depression both independently contributed to mental HRQOL [23, 25]. A population-based study of war veterans found that those who screened positive for PTSD reported a mean of 20 physical health symptoms compared with a mean of four symptoms among those without PTSD [24]. In the same study, $58 \%$ of those with PTSD rated their general health status as poor or fair while only $6 \%$ of those without PTSD rated health status as poor or fair [24]. Persistent PTSD symptoms measured at two discrete time points over a 14-year period among Vietnam veterans were found to be associated with worse family relationships, less life satisfaction and happiness, more mental health service use, and more non-specific health complaints than those without [26]. PTSD often has a duration of many years [7], and is characterized by frequent comorbid mental and physical health conditions among 9/11 exposed populations [5, 13, 15-18]. It is essential to understand how the long-term course of 9/11-related PTSD 10 years post-disaster influences HRQOL based on more frequent follow-up data.

The 9/11 exposed individuals with comorbid physical and mental health conditions have worse HRQOL than those without PTSD or with PTSD alone years after the event [5, $17,18]$. A recent study of $9 / 11$ responders reported that persons with active PTSD were 2.6 times more likely and those with remitted PTSD 1.8 times more likely than unaffected responders to report having fair/poor overall health status after adjusting for depression and other covariates [6]. In other previous studies of 9/11-exposed populations, those with PTSD and depression, or those with PTSD and lower respiratory symptoms were more than two times as likely as patients with PTSD alone to report $\geq 14$ unhealthy days in the last 30 days $[5,17]$. However, research examining the extent to which comorbid depression, anxiety, and chronic physical conditions affect the association between PTSD trajectory and HRQOL has been limited.

This report addresses two objectives: (1) to estimate the burden of medical morbidity by 9/11-related PTSD trajectory group a decade after $9 / 11$; and (2) to estimate the impact of physical and mental health comorbidities on the association between 9/11-related PTSD trajectories and HRQOL. In this study we hypothesized that the prevalence of comorbid depression and/or anxiety, as well as comorbid physical health conditions would be higher among those who had more years of PTSD symptoms than those who had fewer years of symptoms. Individuals with PTSD who have comorbid psychological and physical health conditions would have significantly worse quality of life 10 years after the $9 / 11$ disaster than those without comorbidity.

\section{Methods}

\section{Study sample}

Data came from the WTC Health Registry ("Registry"), a longitudinal closed cohort study created in 2002 as a public health response to 9/11 [27]. A total of 71,431 individuals who lived, worked, or went to school in the area of the WTC disaster on 9/11, or were involved in rescue and recovery work were enrolled on a voluntary basis in 2003-2004 (Wave 1, W1) [27]. Enrollees were either identified through lists provided by employers, government agencies, and other entities (30\%), or they responded to an outreach campaign (70\%). This study used data from W1 and two follow-up surveys conducted in 2006-2007 (Wave 2, W2) and 2011-2012 (Wave $3, \mathrm{~W} 3$ ). Of the 68,672 people aged $\geq 18$ years on $9 / 11 / 2001$ who enrolled in the Registry (W1), 35,857 
$(52.5 \%)$ participated in the next two follow-up surveys (W2 and W3). After excluding proxy responses $(n=309)$, enrollees reporting physician-diagnosed pre-9/11 PTSD $(n=497)$, enrollees missing items on the self-reported PTSD Checklist (PCL) assessment at any wave, or missing values on HRQOL measures, depression, and anxiety assessments at W3 ( $n=5049)$, the final sample was 30,002.

The Centers for Disease Control and Prevention (CDC) and the NYC Department of Health and Mental Hygiene institutional review boards approved the World Trade Center Health Registry protocol.

\section{Measures}

\section{Health-related quality of life (HRQOL)}

HRQOL, the outcome of interest, was assessed at W3 using the CDC HRQOL 4-item Healthy Days Measure (CDC HRQOL-4) [28, 29]. The CDC HRQOL-4 assesses an individual's perceived sense of well-being through four questions: (1) Would you say that in general your health is excellent, very good, good, fair, or poor?; (2) Thinking about your physical health, which includes physical illness and injury, for how many days during the last 30 days was your physical health not good?; (3) Thinking about your mental health, which includes stress, depression, and problems with emotions, for how many days during the last 30 days was your mental health not good?; and (4) For about how many days did poor physical or mental health keep you from doing your usual activities during the last 30 days? These HRQOL measures have been shown to have good validity and reliability [28, 29].

Based on response to above questions, three indicators of poor HRQOL were derived for use in this study to assess different aspects of HRQOL [28]: (1) general health status, dichotomized into poor or fair vs. good, very good, or excellent, to signify overall self-rated health; (2) unhealthy days (the sum of physical and mental unhealthy days), with a logical maximum of 30 unhealthy days (Centers for Disease Control and Prevention) ( $\geq 14$ vs. $<14$ days), to measure recent physical symptoms and/or mental distress ; and (3) activity limitation days ( $\geq 14$ vs. $<14$ days), to indicate perceived disability as well as lower productivity [28, 29].

\section{Post-9/11 PTSD trajectory}

Post-9/11 probable PTSD trajectory was the main predictor of interest. Probable PTSD was assessed at each wave using the PTSD Checklist-Specific (PCL-S), a 17-item self-reported symptom scale, which referred specifically to the events of 9/11. The 17 PCL-S items correspond to the three PTSD symptom clusters (re-experiencing, avoidance, hyperarousal) from the Diagnostic and Statistical
Manual of Mental Disorders (DSM-IV) [30]. The PCL-S is a well-validated measure and has good temporal stability, internal consistency ( $\alpha>0.75)$, test-retest reliability (correlation coefficient, $r=0.66)$, and high convergent validity $(r=0.58-0.93)$ [31]. Enrollees were asked to rate the degree to which they were bothered by symptoms in the past 30 days from 1 (not at all) to 5 (extremely). Responses to the 17 items were summed, for a total score of 17 to 85 . Probable PTSD (subsequently referred to as PTSD) was defined as a PCL-S score $\geq 44$ (overall diagnostic efficiency $=0.90$, sensitivity $=0.94$, and specificity $=0.86$ ) [32], with at least one re-experiencing symptom (DSM-IV criterion B), three avoidance symptoms (DSM-IV criterion C), and two hyperarousal symptoms (DSM-IV criterion D) [33]. In the present sample, the internal consistency was excellent (Cronbach's alpha $=0.95$ ). Enrollees were further categorized into four trajectory groups based on their PTSD status across waves: (1) chronic (W1+ and W3+); (2) delayed (W1- and W3+); (3) remitted ((W1+ or W2+) and W3-); and (4) no PTSD (W1- and W2- and W3-).

\section{Other covariates}

The main confounders included in multivariate analyses were mental health (depression and/or anxiety, referred to as depression/anxiety) and physical health comorbidity. Current depression was measured at W3 using the 8-item Patient Health Questionnaire (PHQ-8) and defined as a PHQ-8 score $\geq 10$ (positive predictive value $=96.5 \%$ ) [34]. The PHQ-8 consists of eight out of nine criteria on which a DSM-IV depression diagnosis is based [30]. Anxiety was measured at W3 using the 7-item Generalized Anxiety Disorder Scale (GAD-7) and defined as a GAD-7 score $\geq 10$ (internal consistency, $\alpha=0.92$; intra-class correlation $=0.83$ ) [35]. Both the PHQ-8 and GAD-7 ask enrollees to rate how often they were bothered by a symptom during the last 2 weeks on a 4-point scale from 0 (not at all) to 3 (nearly every day).

Physical health comorbidity was defined based on selfreport of any of 18 physician-diagnosed medical conditions at W3. These conditions included respiratory problems (asthma, reactive airways dysfunction syndrome (RADS), emphysema, chronic bronchitis, pulmonary fibrosis, or asbestosis), cardiovascular problems (hypertension, heart attack, angina, coronary heart disease, or stroke), autoimmune and endocrine problems (diabetes, rheumatoid arthritis, multiple sclerosis, amyotrophic lateral sclerosis, lupus, scleroderma, polymyositis, or thyroid), gastroesophageal reflux disease, and sarcoidosis.

Other covariates included in the multivariate analyses were sociodemographic characteristics, Registry enrollee eligibility group (rescue/recovery workers and volunteers vs. community members), and social support. 
Sociodemographic characteristics included age, gender, race/ethnicity, body mass index (BMI), physical activity, smoking history (current, former, never), household income ( $<\$ 75,000$ vs. $\geq \$ 75,000)$, and employment status (unable to work due to poor health, retired, unemployed/ other, employed). A social support measure covering multiple dimensions (e.g., emotional and tangible support) was assessed at W3. Respondents rated five items, including whether someone was available when needed to take them to the doctor, have a good time with, hug them, prepare meals, and understand their problems using a five-point scale from none (0) to all (4) of the time. The five items were summed, with a total score ranging from 0 to 20 . The total score was then used to create a four-level social support variable categorized as none or little (0-5), some (6-10), most (11-15), or all (16-20) of the time.

\section{Statistical analysis}

We first described the study population using frequency and proportions. We then computed age-adjusted (2000 U.S. Standard Population) prevalence of overall and individual comorbidity for all studied individuals and by 4-level PTSD trajectory using SAS-callable SUDAAN. Overall comorbidity was defined based on the presence of any depression or anxiety and any of the physical health conditions and categorized into 4 groups: comorbid physical and mental conditions, comorbid depression and/or anxiety alone, any comorbid physical condition alone, and no comorbidity. To examine the association between PTSD trajectory and each of the three indicators of poor HRQOL, we used modified Poisson regression with a robust error variance [36] to estimate the prevalence ratios directly. In multivariate analyses, we assessed the independent association between PTSD trajectory and each of the HRQOL indicators, respectively, in two separate analyses. Analysis 1 did not include physical and mental health comorbidity, and Analysis 2 included comorbidity, both analyses adjusted for sociodemographic characteristics, Registry eligibility group, and social support. In Analysis 2, comorbidity was represented as a 16-level categorical independent variable which combined PTSD trajectory and overall comorbidity status; the no-PTSD group with no comorbidity was the referent.

\section{Sensitivity analysis}

Knowing that W3 non-participants were more likely to be younger, less educated, to have lower household income, and higher prevalence of 9/11-related PTSD compared to W3 participants [37], we conducted a sensitivity analysis to explore the potential impact of selection bias resulting from loss to follow-up. Specifically, we repeated Analysis 2 among W3 non-participants who participated in W2 and met similar inclusion criteria such as age $\geq 18$ years on $9 / 11$ and no pre-9/11 PTSD (Supplementary Table 1). Due to lack of PHQ-8 and GAD-7 data at W2, we instead used selfreported physician-diagnosed depression and anxiety. Additionally, information on some physical health conditions and social support was not available for the sensitivity analysis.

All analyses were performed in SAS Version 9.4 (SAS Institute, Inc., Cary, NC). Statistical significance was set at a two-sided 0.05 alpha level.

\section{Results}

Table 1 describes the sociodemographic characteristics of the study sample $(n=30,002)$ at $\mathrm{W} 3$. The study enrollees were predominantly $45-64$ years old $(61.6 \%)$, male $(63.0 \%)$, non-Hispanic white $(74.2 \%)$, employed $(69.0 \%)$, never smokers $(56.1 \%)$, physically active $(75.4 \%)$, overweight or obese $(70.7 \%)$, community members $(51.7 \%)$, had an annual household income $\geq \$ 75,000(60.4 \%)$, and frequent social support $(76.4 \%)$. Most $(65.6 \%)$ enrollees reported at least one of the 18 physical health conditions, and $19.0 \%$ had depression or anxiety at W3.

Age-adjusted prevalence of overall comorbidity by PTSD trajectory is depicted in Fig. 1. The age-adjusted prevalence of comorbid physical and mental health conditions was highest in the chronic PTSD (68.6\%), followed by the delayed $(57.2 \%)$, remitted $(23.8 \%)$, and no-PTSD groups $(5.1 \%)$. In contrast, the age-adjusted prevalence of no comorbidity was highest in the no-PTSD group (38.6\%), and lowest in the chronic PTSD group (4.2\%).

Age-adjusted prevalence of individual comorbidity in the study sample is shown in Table 2 . The age-adjusted prevalence was $14.9 \%$ for depression and $12.1 \%$ for anxiety. Hypertension (32.3\%), gastroesophageal reflux disease (21.4\%), and asthma (19.6\%) were the physical conditions with the highest age-adjusted prevalence. When stratified by PTSD trajectory, the prevalence of each of the mental or physical health conditions listed except for coronary heart disease and heart attack was highest for the chronic PTSD, followed by delayed PTSD, remitted PTSD, and the lowest for the no-PTSD group. The prevalence ratio of chronic to no-PTSD groups ranged from 1.4 for hypertension to 15.1 for anxiety (Table 2).

Overall, $23.5 \%$ of enrollees reported having poor or fair health status, $32.3 \%$ reported $\geq 14$ unhealthy days, and $11.9 \%$ reported $\geq 14$ activity limitation days at W3 (data not shown). The prevalence of each indicator of poor HRQOL was highest among those with chronic PTSD $(67.4 \%$ poor or fair health status, $83.5 \% \geq 14$ unhealthy days, and $48.5 \%$ $\geq 14$ activity limitation days) and lowest among those with no PTSD (15.3\% poor or fair health status, $22.3 \% \geq 14$ unhealthy days, and $6.3 \% \geq 14$ activity limitation days). 
Table 1 Characteristics of study population at Wave $3(N=30,002)$

\begin{tabular}{|c|c|c|}
\hline & $N$ & $(\%)^{\mathrm{a}}$ \\
\hline \multicolumn{3}{|l|}{ Age, years } \\
\hline $25-44$ & 7671 & $(25.6)$ \\
\hline $45-64$ & 18,469 & $(61.6)$ \\
\hline$\geq 65$ & 3862 & $(12.9)$ \\
\hline \multicolumn{3}{|l|}{ Gender } \\
\hline Male & 18,892 & $(63.0)$ \\
\hline Female & 11,110 & $(37.0)$ \\
\hline \multicolumn{3}{|l|}{ Race/ethnicity } \\
\hline Non-Hispanic white & 22,263 & $(74.2)$ \\
\hline Non-Hispanic black & 2589 & (8.6) \\
\hline Hispanic & 2996 & $(10.0)$ \\
\hline Asian and others & 2154 & $(7.2)$ \\
\hline \multicolumn{3}{|l|}{ Annual household income in $2010, \$$} \\
\hline$<75,000$ & 10,538 & $(35.1)$ \\
\hline$\geq 75,000$ & 18,132 & $(60.4)$ \\
\hline \multicolumn{3}{|l|}{ Employment status } \\
\hline Unable to work due to health & 1834 & $(6.1)$ \\
\hline Retired & 5296 & $(17.7)$ \\
\hline Unemployed and other & 2072 & $(6.9)$ \\
\hline Employed & 20,710 & $(69.0)$ \\
\hline \multicolumn{3}{|l|}{ Smoking status } \\
\hline Current & 3012 & $(10.0)$ \\
\hline Former & 10,081 & $(33.6)$ \\
\hline Never & 16,830 & $(56.1)$ \\
\hline \multicolumn{3}{|l|}{ Physical inactivity during last month } \\
\hline Yes & 7229 & $(24.1)$ \\
\hline No & 22,633 & $(75.4)$ \\
\hline \multicolumn{3}{|l|}{ Body mass index (BMI) } \\
\hline$\geq 30($ Obese $)$ & 9562 & $(31.9)$ \\
\hline 25 to $<30$ (Overweight) & 11,632 & $(38.8)$ \\
\hline$<25$ (Normal/underweight) & 8396 & $(28.0)$ \\
\hline \multicolumn{3}{|l|}{ Perceived social support } \\
\hline None-to-little & 2565 & $(8.5)$ \\
\hline Some of the time & 4477 & $(14.9)$ \\
\hline Most of the time & 8466 & $(28.2)$ \\
\hline All of the time & 14,457 & $(48.2)$ \\
\hline \multicolumn{3}{|l|}{ Registry enrollees } \\
\hline Rescue/recovery workers & 14,488 & $(48.3)$ \\
\hline Community members & 15,514 & $(51.7)$ \\
\hline \multicolumn{3}{|l|}{ PTSD trajectory } \\
\hline Chronic & 1749 & $(5.8)$ \\
\hline Delayed & 2139 & $(7.1)$ \\
\hline Remitted & 2901 & $(9.7)$ \\
\hline No PTSD & 23,213 & $(77.7)$ \\
\hline Ever reported physical health condition & 19,691 & $(65.6)$ \\
\hline Depression or anxiety & 5709 & $(19.0)$ \\
\hline
\end{tabular}

PTSD posttraumatic stress disorder

${ }^{a}$ May not sum to $100 \%$ due to missing values
When stratified by overall comorbidity status, the prevalence of each of the three indicators of poor HRQOL decreased as PTSD improved. For each PTSD trajectory group, those with physical and mental health comorbidity were most likely to report poor HRQOL and those with no comorbidities were least likely.

In multivariate analyses, adjusting for socio-demographics, Registry eligibility group, and social support (Table 3), PTSD trajectory was independently associated with each of the three indicators of poor HRQOL. In Analysis 1 without accounting for comorbidity, the adjusted prevalence ratio (APR) for each indicator of poor HRQOL was truncated but remained significant, with an APR ranging from 1.8 to 3.3. Individuals with chronic PTSD had elevated risk of poor or fair health status (APR 2.2; 95\% CI 2.1, 2.3), $\geq 14$ unhealthy days (APR 2.3; 95\% CI 2.2, 2.4), and $\geq 14$ activity limitation days (APR 3.3; 95\% CI 3.1, 3.7) compared to the no-PTSD group. Similar associations were observed for delayed PTSD.

When physical and mental health comorbidity status was accounted for in Analysis 2 (Table 3), the APR increased to 7.3 for poor or fair health status (95\% CI 6.5, 8.2), 4.7 for $\geq 14$ unhealthy days (95\% CI 4.4, 5.1), and 9.6 for $\geq 14$ activity limitation days (95\% CI 8.1, 11.4) in those with chronic PTSD who also had comorbid physical and mental health conditions compared to the no-PTSD group that had no comorbidity. The highest risk for poor HRQOL was observed among those with both physical and mental health conditions, followed by comorbid mental health conditions alone and comorbid physical health conditions alone; the lowest risk was found among those with no comorbidity. Similar risk estimates for poor HRQOL were also observed among those with delayed PTSD.

In conducting the sensitivity analysis, we found attenuated but significant associations between PTSD trajectory and each of the three HRQOL indicators, when accounting for physical and mental health comorbidity. The APR was 5.5 for poor or fair health status ( $95 \%$ CI 4.7, 6.5), 4.3 for $\geq 14$ unhealthy days (95\% CI 3.9, 4.8), and 8.3 for $\geq 14$ activity limitation days (95\% CI 6.5, 10.6) for individuals with chronic PTSD who also had comorbid physical and mental health conditions compared to the no-PTSD group that had no comorbidity (Supplementary Table 1).

\section{Discussion}

More than a decade after $9 / 11$, a substantial proportion of a cohort of persons exposed to the $9 / 11$ disaster reported significant physical and mental health comorbidity and diminished HRQOL, particularly among those with PTSD. The results of this study can be best summarized through three main findings. First, the physical and mental health burden 
Fig. 1 Age-adjusted prevalence of overall comorbidity status by PTSD trajectory

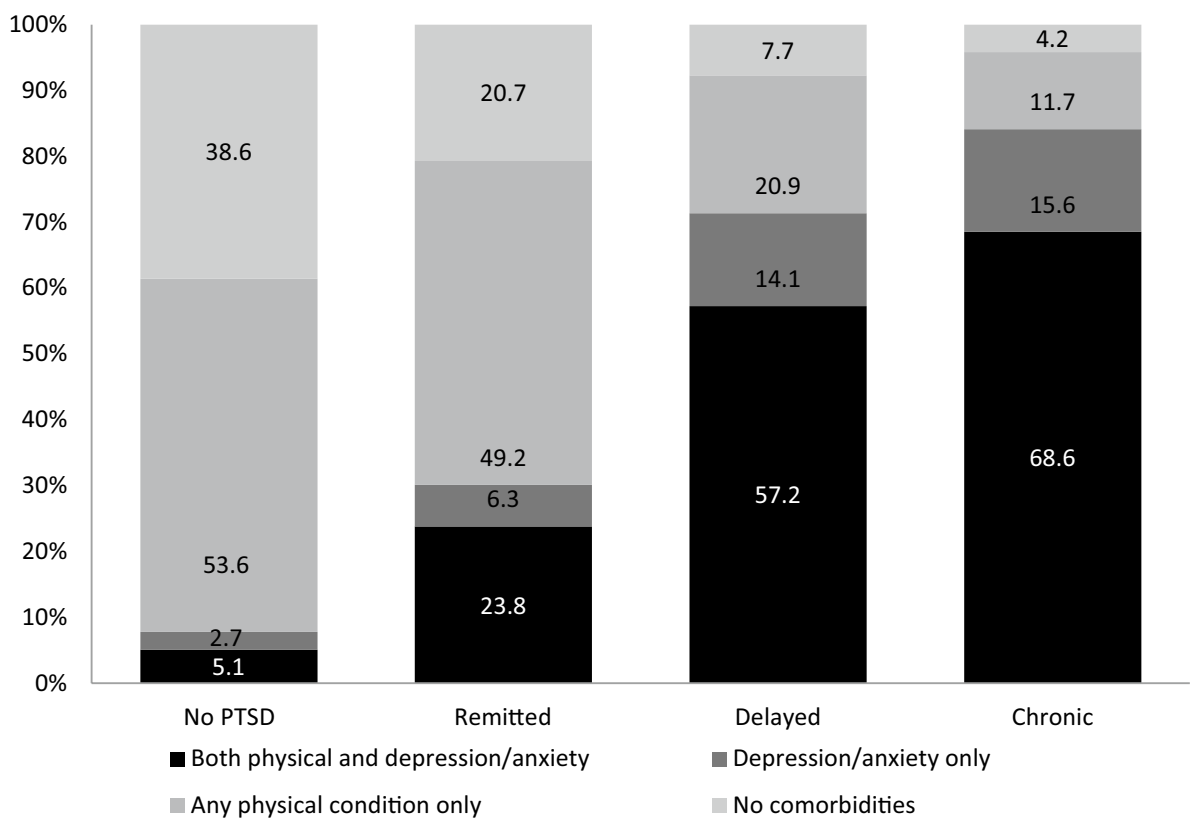

Table 2 Age-adjusted prevalence of physical and mental health comorbidity by PTSD trajectory $(N=30,002)^{\mathrm{a}}$

\begin{tabular}{|c|c|c|c|c|c|c|}
\hline & \multirow[t]{2}{*}{ All } & \multicolumn{4}{|c|}{ PTSD trajectory } & \multirow{2}{*}{$\begin{array}{l}\text { Prevalence ratio } \\
\text { (chronic/no PTSD) }\end{array}$} \\
\hline & & Chronic & Delayed & Remitted & No PTSD & \\
\hline \multicolumn{7}{|l|}{ Mental health condition at Wave 3} \\
\hline Depression (PHQ-8 score $\geq 10$ ) & 14.9 & 77.2 & 64.0 & 23.4 & 5.5 & 14.0 \\
\hline Anxiety (GAD-7 score $\geq 10$ ) & 12.1 & 66.4 & 50.9 & 18.3 & 4.4 & 15.1 \\
\hline \multicolumn{7}{|l|}{ Ever reported physical health condition } \\
\hline Gastroesophageal reflux disease & 21.4 & 39.4 & 36.0 & 27.0 & 18.2 & 2.2 \\
\hline Sarcoidosis & 1.0 & 1.9 & 1.8 & 1.4 & 0.9 & 2.1 \\
\hline \multicolumn{7}{|l|}{ Respiratory problem } \\
\hline Asbestosis & 0.6 & 2.0 & 1.6 & 0.6 & 0.4 & 5.0 \\
\hline Asthma & 19.6 & 35.0 & 29.8 & 26.7 & 16.9 & 2.1 \\
\hline Chronic bronchitis & 8.7 & 23.0 & 20.7 & 13.9 & 6.2 & 3.7 \\
\hline Emphysema & 4.2 & 10.8 & 10.4 & 5.5 & 3.1 & 3.5 \\
\hline Pulmonary fibrosis & 0.8 & 3.4 & 2.0 & 1.4 & 0.5 & 6.8 \\
\hline Reactive airway dysfunction syndrome or RADS & 3.6 & 10.7 & 9.5 & 5.1 & 2.4 & 4.5 \\
\hline \multicolumn{7}{|l|}{ Cardiovascular problem } \\
\hline Angina & 2.4 & 6.4 & 4.2 & 3.0 & 1.9 & 3.4 \\
\hline Coronary heart disease & 4.6 & 7.3 & 7.8 & 5.0 & 4.1 & 1.8 \\
\hline Heart attack & 2.7 & 4.6 & 4.9 & 3.4 & 2.4 & 1.9 \\
\hline Hypertension & 32.3 & 43.7 & 41.4 & 37.1 & 30.2 & 1.4 \\
\hline Stroke & 1.5 & 3.0 & 2.5 & 1.9 & 1.3 & 2.3 \\
\hline \multicolumn{7}{|l|}{ Autoimmune and endocrine disorders } \\
\hline Diabetes & 8.7 & 15.6 & 11.9 & 11.9 & 7.6 & 2.1 \\
\hline Multiple sclerosis or amyotrophic lateral sclerosis & 0.6 & 0.8 & 0.7 & 0.7 & 0.5 & 1.6 \\
\hline Rheumatoid arthritis & 6.0 & 15.1 & 10.7 & 9.5 & 4.6 & 3.3 \\
\hline $\begin{array}{l}\text { Other autoimmune disorders (e.g., lupus, sclero- } \\
\text { derma, polymyositis) }\end{array}$ & 2.7 & 6.0 & 4.3 & 3.4 & 2.3 & 2.6 \\
\hline Thyroid disease & 7.9 & 12.1 & 10.8 & 9.5 & 7.2 & 1.7 \\
\hline
\end{tabular}

PTSD posttraumatic stress disorder

${ }^{\text {a }}$ They are not mutually exclusive 
Table 3 Adjusted prevalence ratio (APR) of PTSD trajectory with poor HRQOL by physical and mental comorbidity among adult WTCHR enrollees $(N=30,002)$

\begin{tabular}{|c|c|c|c|c|}
\hline & \multirow[t]{2}{*}{$N$} & \multicolumn{3}{|c|}{$\operatorname{APR}(95 \% \text { confidence interval })^{\mathrm{a}}$} \\
\hline & & Fair/poor general health & $\geq 14$ Unhealthy days ${ }^{b}$ & $\begin{array}{l}\geq 14 \text { Activity } \\
\text { limitation } \\
\text { days }^{b}\end{array}$ \\
\hline \multicolumn{5}{|l|}{ Analysis $1^{\mathrm{c}}$} \\
\hline \multicolumn{5}{|c|}{ Probable PTSD at Wave 3} \\
\hline Chronic & 1749 & $2.2(2.1-2.3)$ & $2.3(2.2-2.4)$ & $3.3(3.1-3.7)$ \\
\hline Delayed & 2139 & $2.2(2.1-2.3)$ & $2.3(2.3-2.5)$ & $3.1(2.9-3.4)$ \\
\hline Remitted & 2901 & $1.8(1.7-1.9)$ & $1.8(1.7-1.9)$ & $1.8(1.6-2.0)$ \\
\hline No PTSD & 23,213 & Referent & Referent & Referent \\
\hline \multicolumn{5}{|l|}{ Analysis $2^{\mathrm{d}}$} \\
\hline \multicolumn{5}{|l|}{ Comorbid both $^{\mathrm{e}}$} \\
\hline Chronic PTSD & 1240 & $7.3(6.5-8.2)$ & $4.7(4.4-5.1)$ & $9.6(8.1-11.4)$ \\
\hline Delayed PTSD & 1270 & $7.3(6.5-8.2)$ & $4.8(4.5-5.2)$ & $9.1(7.7-10.7)$ \\
\hline Remitted PTSD & 719 & $7.1(6.3-8.1)$ & $4.7(4.3-5.1)$ & $7.3(6.1-8.8)$ \\
\hline No PTSD & 1265 & $6.3(5.6-7.1)$ & $4.6(4.3-4.9)$ & $7.1(6.0-8.4)$ \\
\hline \multicolumn{5}{|c|}{ Comorbid depression/anxiety only } \\
\hline Chronic & 227 & $6.4(5.5-7.5)$ & $5.1(4.6-5.6)$ & $8.8(7.0-11.1)$ \\
\hline Delayed & 273 & $6.0(5.1-7.1)$ & $5.2(4.8-5.7)$ & $8.8(7.0-11.1)$ \\
\hline Remitted & 173 & $4.5(3.5-5.8)$ & $4.3(3.8-4.9)$ & $5.0(3.4-7.4)$ \\
\hline No PTSD & 538 & $3.9(3.3-4.7)$ & $4.4(4.0-4.9)$ & $5.1(3.9-6.5)$ \\
\hline \multicolumn{5}{|c|}{ Comorbid physical condition only } \\
\hline Chronic & 211 & $6.8(5.7-8.0)$ & $3.9(3.4-4.4)$ & $5.3(3.9-7.0)$ \\
\hline Delayed & 434 & $6.5(5.7-7.5)$ & $3.8(3.4-4.1)$ & $5.3(4.2-6.7)$ \\
\hline Remitted & 1478 & $5.6(4.9-6.3)$ & $3.1(2.8-3.3)$ & $3.3(2.7-4.1)$ \\
\hline No PTSD & 13,070 & $3.4(3.1-3.8)$ & $1.8(1.7-1.9)$ & $2.2(1.9-2.6)$ \\
\hline \multicolumn{5}{|l|}{ Comorbid neither } \\
\hline Chronic & 71 & $4.1(2.8-6.1)$ & $3.5(2.7-4.5)$ & $3.4(1.8-6.8)$ \\
\hline Delayed & 158 & $4.5(3.3-6.1)$ & $3.1(2.6-3.8)$ & $3.8(2.4-6.0)$ \\
\hline Remitted & 531 & $2.6(2.0-3.3)$ & $2.1(1.8-2.4)$ & $1.8(1.2-2.8)$ \\
\hline No PTSD & 8340 & Referent & Referent & Referent \\
\hline
\end{tabular}

PTSD posttraumatic stress disorder

${ }^{a}$ Adjusted for sociodemographic characteristics (age, gender, race, income, employment status, physical activity, BMI, smoking status), Registry eligibility group and social support

${ }^{b} \geq 14$ unhealthy or $\geq 14$ activity limitation days in the last 30 days

${ }^{c}$ The multivariate analyses did not include physical and mental health comorbidity

${ }^{\mathrm{d}}$ The multivariate analyses included physical and mental health comorbidity

${ }^{\mathrm{e}}$ Comorbid both depression/anxiety at Wave 3 and any of ever reported physical health conditions among those with PTSD is striking and worsens over the period of chronic PTSD symptoms. Second, active PTSD (chronic and delayed), regardless of the duration, was significantly associated with each of three indicators of poor HRQOL, independent of sociodemographic characteristics, social support, and comorbidity. Third, the magnitude of the effect of PTSD trajectory on each of the HRQOL indicators varied depending on the presence of physical and mental health comorbidity after adjusting for covariates.

The physical and mental health burden among 9/11-exposed individuals was significant years after exposure, particularly among those with chronic PTSD. Of numerous physical and mental health conditions examined, the age-adjusted prevalence among the entire study population was higher than in the general population for a number of conditions: depression (14.9 vs. 8.2\%) [38], asthma (19.6 vs. $8.4 \%$ ) [39], chronic bronchitis (8.7 vs. $4.4 \%$ ) [40], and pulmonary fibrosis (0.8 vs. $0.04-0.06 \%)$ [41]. When ageadjusted prevalence of health conditions was stratified by PTSD trajectory, enrollees with chronic PTSD were 14 to 15 times more likely to have depression or anxiety at W3, and 1.4 to 6.8 times more likely to have ever reported physical health conditions (1.4 for hypertension and 6.8 times for pulmonary fibrosis) than those with no PTSD. The higher 
prevalence of physical and mental health conditions in this study is consistent with the existing 9/11 literature documenting the impact of exposure on adverse health outcomes.

Early data from the National Comorbidity Survey revealed that more than one-third of individuals with PTSD failed to recover, even after many years [7]. Similarly, onethird of our study population remained symptomatic over a nearly 10-year period of follow-up. Consistent with other studies [6, 19], we also found that PTSD symptoms were significantly associated with impaired HRQOL.

However, the effect of PTSD trajectory on HRQOL was largely impacted by physical or mental health comorbidities in this study. Depression/anxiety and physical health conditions amplify the adverse effect of PTSD symptoms on quality of life. Those with chronic or delayed PTSD and comorbid physical and mental health conditions experienced the greatest decrement in HRQOL, followed by those with a mental or physical health condition alone. Chronic and delayed PTSD did not appear to be differentially associated with either of the poor HRQOL indicators; this suggests that in the presence of PTSD, HRQOL may be more strongly influenced by comorbid conditions than the duration of PTSD. Notably, the comorbid depression/anxiety has a greater impact on the PTSD-HRQOL (specifically, unhealthy days and activity limitation days) associations than comorbid physical health condition only. These highrisk groups may have more severe health problems or conditions that are not being well managed or treated. Understanding and mitigating the impact of the co-occurrence of two or more health conditions on PTSD trajectory and poor HRQOL has important implications for treatment planning and future disaster preparedness and response.

One of the HRQOL impairment indicators, activity limitation, emerged as an important issue in the population studied. Frequent limited activity is a major source of health and economic burden at both the individual and societal level $[42,43]$. In the present study, individuals with chronic and delayed PTSD and comorbid physical and mental health conditions experienced nine times the risk of activity limitation ( $\geq 14$ in the last 30 days) compared to those without PTSD and comorbidity, further highlighting the debilitating effects of comorbidity. A previous study using Registry data found that co-occurring PTSD and respiratory illness was significantly associated with premature labor force exit and lower income [44]. As such, our findings may have broad public health and economic implications. It is important to understand the magnitude of comorbidity's impact on the HRQOL of those with PTSD, and to account for multiple comorbidities when providing clinical care to patients with PTSD. This also underscores the importance of integration of physical and mental health care.

Research has shown that improvement in PTSD symptoms can result in better HRQOL [6]. In this study, improved
HRQOL (reduced poor or fair health status and decreased unhealthy days) among those with remitted PTSD was observed in those with comorbid mental or physical health conditions alone and in those with neither comorbidity, but not in those with both mental and physical comorbid conditions. Thus, multiple comorbidities are an important factor in HRQOL impairment among individuals with remitted PTSD.

There are several strengths to this study. This is the first 9/11 study to assess the association between PTSD trajectory and HRQOL, and how the magnitude of the association changes in the presence of physical and/or mental health comorbidity 10-11 years after 9/11. In assessing comorbidity, this study examined numerous physical and mental health conditions, including depression, anxiety, and 18 physician-diagnosed physical health conditions. Furthermore, the study population included both first responders and survivors from the affected area, broadening the external validity of the findings. Lastly, the large post-disaster population and availability of important demographic covariates and other HRQOL risk factors offer sufficient power to examine the association between post-9/11 PTSD trajectory and HRQOL.

The findings in this study are subject to some limitations. First, our findings may be distorted by potential selection bias resulting from loss to follow-up if attrition is related to both the exposure and outcome under study [45, 46]. The results of sensitivity analysis on the associations between PTSD trajectory and HRQOL, accounting for comorbid physical and mental health conditions, were significant and in the same direction as in our complete case analysis. But the results among W3 non-participants (Supplementary Table 1) were attenuated, suggesting attrition may have influenced the results and that those lost to follow-up may be healthier than those who chose to stay in the study. A second limitation is the reliance on self-report to identify mental and physical health conditions. However, the use of validated, standardized questionnaire measures to identify mental disorders (i.e., PTSD, depression, and anxiety) and HRQOL may help to minimize potential reporting bias. Lastly, lack of clinical information on treatment (e.g., type and duration of medication) impeded our ability to control for these variables in the analysis.

\section{Conclusions}

This study quantifies the extent to which comorbid depression, anxiety, and chronic physical conditions affect the association between 9/11-related PTSD trajectories and HRQOL. Our findings have implications for both PTSD researchers and clinicians. The substantial psychological and physical comorbidity in chronic PTSD patients and its contribution 
to a greater decrement of HRQOL years after the WTC exposure is a cause for longer-term public health concern. Early and repeated screening for PTSD and comorbid health conditions among those exposed to disaster or trauma is an important component of future disaster response and followup in order to provide services to affected individuals and an opportunity to prevent and reduce comorbidity. In short, optimizing functioning and improving HRQOL through integration of physical and mental health care at both the individual and community level may be an important target in the early identification and treatment of PTSD and comorbidity.

Acknowledgements We gratefully acknowledge the participation of all Registry enrollees. We thank Drs. Charon Gwynn, James Hadler, and Lisa Gargano for their helpful comments.

Author contributions All authors made substantial contributions to the conception or design of the work, or the acquisition, analysis, or interpretation of data for the work; drafted the work or revised it critically for important intellectual content; provided final approval of the version to be published; and agreed to be accountable for all aspects of the work in ensuring that questions related to the accuracy or integrity of any part of the work are appropriately investigated and resolved.

Funding This publication was supported by Cooperative Agreement Numbers 2U50/OH009739 and 5U50/OH009739 from the National Institute for Occupational Safety and Health (NIOSH) of the Centers for Disease Control and Prevention (CDC); U50/ATU272750 from the Agency for Toxic Substances and Disease Registry (ATSDR), CDC, which included support from the National Center for Environmental Health, CDC; and by the New York City Department of Health and Mental Hygiene (NYC DOHMH). Its contents are solely the responsibility of the authors and do not necessarily represent the official views of NIOSH, CDC, or the Department of Health and Human Services.

\section{Compliance with ethical standards}

Conflict of interest The authors declare that they have no conflict of interest.

Ethical approval The Centers for Disease Control and Prevention (CDC) and the NYC Department of Health and Mental Hygiene institutional review boards approved the World Trade Center Health Registry protocol.

Open Access This article is distributed under the terms of the Creative Commons Attribution 4.0 International License (http://creativecommons.org/licenses/by/4.0/), which permits unrestricted use, distribution, and reproduction in any medium, provided you give appropriate credit to the original author(s) and the source, provide a link to the Creative Commons license, and indicate if changes were made.

\section{References}

1. DiGrande, L., Perrin, M. A., Thorpe, L. E., Thalji, L., Murphy, J., Wu, D., et al. (2008). Posttraumatic stress symptoms, PTSD, and risk factors among lower Manhattan residents 2-3 years after the
September 11, 2001 terrorist attacks. Journal of Traumatic Stress, 21(3), 264-273.

2. Stellman, J. M., Smith, R. P., Katz, C. L., Sharma, V., Charney, D. S., Herbert, R., et al. (2008). Enduring mental health morbidity and social function impairment in world trade center rescue, recovery, and cleanup workers: the psychological dimension of an environmental health disaster. Environmental Health Perspectives, 116(9), 1248-1253.

3. Brackbill, R. M., Hadler, J. L., DiGrande, L., Ekenga, C. C., Farfel, M. R., Friedman, S., et al. (2009). Asthma and posttraumatic stress symptoms 5 to 6 years following exposure to the World Trade Center terrorist attack. JAMA, 302(5), 502-516.

4. Berninger, A., Webber, M. P., Niles, J. K., Gustave, J., Lee, R., Cohen, H. W., et al. (2010). Longitudinal study of probable posttraumatic stress disorder in firefighters exposed to the World Trade Center disaster. American Journal of Industrial Medicine, 53(12), 1177-1185.

5. Caramanica, K., Brackbill, R. M., Liao, T., \& Stellman, S. D. (2014). Comorbidity of 9/11-related PTSD and depression in the World Trade Center Health Registry 10-11 years post disaster. Journal of Traumatic Stress, 27(6), 680-688.

6. Bromet, E. J., Hobbs, M. J., Clouston, S. A., Gonzalez, A., Kotov, R., \& Luft, B. J. (2016). DSM-IV post-traumatic stress disorder among World Trade Center responders 11-13 years after the disaster of 11 September 2001 (9/11). Psychological Medicine, 46(4), 771-783.

7. Kessler, R. C. (2000). Posttraumatic stress disorder: the burden to the individual and to society. The Journal of Clinical Psychiatry, 61(Suppl 5), 4-12.

8. Pietrzak, R. H., Feder, A., Singh, R., Schechter, C. B., Bromet, E. J., Katz, C. L., et al. (2014). Trajectories of PTSD risk and resilience in World Trade Center responders: an 8-year prospective cohort study. Psychological Medicine, 44(1), 205-219.

9. Maslow, C. B., Caramanica, K., Welch, A. E., Stellman, S. D., Brackbill, R. M., \& Farfel, M. R. (2015). Trajectories of scores on a screening instrument for PTSD among World Trade Center rescue, recovery, and clean-up workers. Journal of Traumatic Stress, 28(3), 198-205.

10. Welch, A. E., Caramanica, K., Maslow, C. B., Brackbill, R. M., Stellman, S. D., \& Farfel, M. R. (2016). Trajectories of PTSD among lower Manhattan residents and area workers following the 2001 World Trade Center disaster, 2003-2012. Journal of Traumatic Stress, 29(2), 158-166.

11. Kessler, R. C., Sonnega, A., Bromet, E., Hughes, M., \& Nelson, C. B. (1995). Posttraumatic stress disorder in the National Comorbidity Survey. Archives of General Psychiatry, 52(12), 1048-1060.

12. Rytwinski, N. K., Scur, M. D., Feeny, N. C., \& Youngstrom, E. A. (2013). The co-occurrence of major depressive disorder among individuals with posttraumatic stress disorder: a meta-analysis. Journal of Traumatic Stress, 26(3), 299-309.

13. Bowler, R. M., Kornblith, E. S., Li, J., Adams, S. W., Gocheva, V. V., Schwarzer, R., et al. (2016). Police officers who responded to 9/11: Comorbidity of PTSD, depression, and anxiety 10-11 years later. American Journal of Industrial Medicine, 59(6), 425-436.

14. Scott, K. M., Lim, C., Al-Hamzawi, A., Alonso, J., Bruffaerts, R., Caldas-de-Almeida, J. M., et al. (2016). Association of mental disorders with subsequent chronic physical conditions. JAMA Psychiatry, 73(2), 150-158.

15. Chiu, S., Niles, J. K., Webber, M. P., Zeig-Owens, R., Gustave, J., Lee, R., et al. (2011). Evaluating risk factors and possible mediation effects in posttraumatic depression and posttraumatic stress disorder comorbidity. Public Health Reports, 126(2), 201-209.

16. Wisnivesky, J. P., Teitelbaum, S. L., Todd, A. C., Boffetta, P., Crane, M., Crowley, L., et al. (2011). Persistence of multiple illnesses in World Trade Center rescue and recovery workers: a cohort study. The Lancet, 378(9794), 888-897. 
17. Nair, H. P., Ekenga, C. C., Cone, J. E., Brackbill, R. M., Farfel, M. R., \& Stellman, S. D. (2012). Co-occurring lower respiratory symptoms and posttraumatic stress disorder 5 to 6 years after the World Trade Center terrorist attack. American Journal of Public Health, 102(10), 1964-1973.

18. Friedman, S. M., Farfel, M. R., Maslow, C., Jordan, H. T., Li, J., Alper, H., et al. (2016). Risk factors for and consequences of persistent lower respiratory symptoms among World Trade Center Health Registrants 10 years after the disaster. Occupational and Environmental Medicine, 73(10), 676-684.

19. Schnurr, P. P., Lunney, C. A., Bovin, M. J., \& Marx, B. P. (2009). Posttraumatic stress disorder and quality of life: extension of findings to veterans of the wars in Iraq and Afghanistan. Clinical Psychology Review, 29(8), 727-735.

20. Gaynes, B. N., Burns, B. J., Tweed, D. L., \& Erickson, P. (2002). Depression and health-related quality of life. The Journal of Nervous and Mental Disease, 190(12), 799-806.

21. Comer, J. S., Blanco, C., Hasin, D. S., Liu, S. M., Grant, B. F., Turner, J. B., \& Olfson, M. (2011). Health-related quality of life across the anxiety disorders: results from the national epidemiologic survey on alcohol and related conditions (NESARC). The Journal of Clinical Psychiatry, 72(1), 43-50.

22. Alonso, J., Ferrer, M., Gandek, B., Ware, J. E., Jr., Aaronson, N. K., Mosconi, P., IQOLA Project Group, et al. (2004). Healthrelated quality of life associated with chronic conditions in eight countries: results from the International Quality of Life Assessment (IQOLA) Project. Quality of Life Research, 13(2), 283-298.

23. Raab, P. A., Mackintosh, M. A., Gros, D. F., \& Morland, L. A. (2015). Impact of comorbid depression on quality of life in male combat Veterans with posttraumatic stress disorder. Journal of Rehabilitation Research and Development, 52(5), 563-576.

24. Barrett, D. H., Doebbeling, C. C., Schwartz, D. A., Voelker, M. D., Falter, K. H., Woolson, R. F., \& Doebbeling, B. N. (2002). Posttraumatic stress disorder and self-reported physical health status among U.S. Military personnel serving during the Gulf War period: a population-based study. Psychosomatics, 43(3), 195-205.

25. Aversa, L. H., Stoddard, J. A., Doran, N. M., Au, S., Chow, B., McFall, M., Saxon, A., \& Baker, D. G. (2012). PTSD and depression as predictors of physical health-related quality of life in tobacco-dependent veterans. Journal of Psychosomatic Research, 73(3), 185-190.

26. Koenen, K. C., Stellman, S. D., Sommer, J. F., \& Stellman, J. M. (2008). Persisting posttraumatic stress disorder symptoms and their relationship to functioning in Vietnam Veterans: A 14-year follow-up. Journal of Traumatic Stress, 21(1), 49-57.

27. Farfel, M., DiGrande, L., Brackbill, R., Prann, A., Cone, J., Friedman, S., et al. (2008). An overview of 9/11 experiences and respiratory and mental health conditions among World Trade Center Health Registry enrollees. Journal of Urban Health, 85, 880-909.

28. Centers for Disease Control and Prevention (CDC). (2000). Measuring healthy days: Population assessment of health-related quality of life. Atlanta: Centers for Disease Control and Prevention.

29. Moriarty, D. G., Zack, M. M., \& Kobau, R. (2003). The Centers for Disease Control and Prevention's Healthy Days Measurespopulation tracking of perceived physical and mental health over time. Health and Quality of Life Outcomes, 1, 37.

30. American Psychiatric Association. (1994). Diagnostic and Statistical Manual of Mental Disorders. (4th edn). Washington, DC: American Psychiatric Association.

31. Wilkins, K. C., Lang, A. J., \& Norman, S. B. (2011). Synthesis of the psychometric properties of the PTSD Checklist (PCL) military, civilian, and specific versions. Depression and Anxiety, 28(7), 596-606.

32. Blanchard, E. B., Jones-Alexander, J., Buckley, T. C., \& Forneris, C. A. (1996). Psychometric properties of the PTSD Checklist (PCL). Behaviour, Research, and Therapy, 34, 669-673.

33. Smith, M. Y., Redd, W., DuHamel, K., Vickberg, S. J., \& Ricketts, P. (1999). Validation of the PTSD Checklist-Civilian Version in survivors of bone marrow transplantation. Journal of Traumatic Stress, 12(3), 485-499.

34. Kroenke, K., Strine, T. W., Spitzer, R. L., Williams, J. B., Berry, J. T., \& Mokdad, A. H. (2009). The PHQ-8 as a measure of current depression in the general population. Journal of Affective Disorders, 114(1-3), 163-173.

35. Spitzer, R. L., Kroenke, K., Williams, J. B., \& Löwe, B. (2006). A brief measure for assessing generalized anxiety disorder: the GAD-7. Archives of Internal Medicine, 166(10), 1092-1097.

36. Zou, G. (2004). A modified Poisson regression approach to prospective studies with binary data. American Journal of Epidemiology, 159, 702-706.

37. Yu, S., Brackbill, R. M., Stellman, S. D., Ghuman, S., \& Farfel, M. R. (2015). Evaluation of non-response bias in a cohort study of World Trade Center terrorist attack survivors. BMC Research Notes, 8, 42.

38. Reeves, W. C., Strine, T. W., Pratt, L. A., Thompson, W., Ahluwalia, I., Dhingra, S. S., Centers for Disease Control and Prevention (CDC), et al. (2011). Mental illness surveillance among adults in the United States. MMWR Suppl, 60(3):1-29.

39. Akinbami, L. J., Moorman, J. E., Bailey, C., Zahran, H. S., King, M., Johnson, C. A., et al. (2012). Trends in asthma prevalence, health care use, and mortality in the United States, 2001-2010. NCHS Data Brief, 94, 1-8.

40. American Lung Association. Trends in COPD (chronic bronchitis and emphysema): morbidity and mortality. (2013). http:// www.lung.org/assets/documents/research/copd-trend-report.pdf. Accessed on 5 October 2017.

41. Nalysnyk, L., Cid-Ruzafa, J., Rotella, P., \& Esser, D. (2012). Incidence and prevalence of idiopathic pulmonary fibrosis: review of the literature. European Respiratory Review, 21(126), 355-361.

42. Merikangas, K. R., Ames, M., Cui, L., Stang, P. E., Ustun, T. B., Von Korff, M., et al. (2007). The impact of comorbidity of mental and physical conditions on role disability in the US adult household population. Archives of General Psychiatry, 64(10), $1180-1188$.

43. McKnight-Eily, L. R., Elam-Evans, L. D., Strine, T. W., Zack, M. M., Perry, G. S., Presley-Cantrell, L., et al (2009). Activity limitation, chronic disease, and comorbid serious psychological distress in U.S. adults-BRFSS 2007. International Journal of Public Health, 54(Suppl 1), 111-119.

44. Yu, S., Brackbill, R. M., Locke, S., Stellman, S. D., \& Gargano, L. M. (2016). Impact of 9/11-related chronic conditions and PTSD comorbidity on early retirement and job loss among World Trade Center disaster rescue and recovery workers. American Journal of Industrial Medicine, 59(9), 731-741.

45. Gustavson, K., von Soest, T., Karevold, E., \& Røysamb, E. (2012). Attrition and generalizability in longitudinal studies: findings from a 15-year population-based study and a Monte Carlo simulation study. BMC Public Health, 12, 918.

46. Rinsky, J. L., Richardson, D. B., Wing, S., Beard, J. D., Alavanja, M., Beane Freeman, L. E., et al. (2017). Assessing the potential for bias from nonresponse to a study follow-up interview: An example from the agricultural health study. American Journal of Epidemiology, 186(4), 395-404. 